\title{
Near-infrared spectroscopy muscle oximetry of patients with postural orthostatic tachycardia syndrome
}

\author{
Parvathi Kadamati*, Jeffrey J. Sugar*, Brendan J. Quirk ${ }^{\dagger}$, Shima Mehrvar*, \\ Gisela G. Chelimsky*, Harry T. Whelan ${ }^{\dagger}$, Thomas C. Chelimsky* \\ and Mahsa Ranji* ${ }^{*}$ \\ *Biophotonics Laboratory \\ University of Wisconsin Milwaukee \\ Department of Electrical Engineering and Computer Science \\ 3200 N Cramer St., Milwaukee, WI 53211, USA \\ ${ }^{\dagger}$ Medical College of Wisconsin \\ Department of Neurology \\ 8701 W Watertown Plank Rd, Milwaukee, WI 53226, USA \\ $\$$ Medical College of Wisconsin \\ Department of Pediatries (Gastroenterology) \\ 8701 W Waterown Plank Rd, Milwaukee, WI 53226, USA

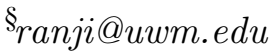

Received 24 January 2018

Accepted 24 June 2018

Published 13 August 2018

\begin{abstract}
Postural orthostatic tachycardia syndrome (POTS) is a disabling condition characterized by orthostatic intolerance with tachycardia in the absence of drop-in blood pressure. A custom-built near-infrared spectroscopy device (NIRS) is applied to monitor the muscle oxygenation, noninvasively in patients undergoing incremental head-up tilt table (HUT). Subjects (6 POTS patients and 6 healthy controls) underwent 30 mins of $70^{\circ}$ on a HUT. The results showed a significant difference in deoxyhemoglobin $(\mathrm{Hb})$, change-in-oxygenation $(\Delta \mathrm{Oxy})$ and blood volume $(\Delta \mathrm{BV})$ between patients and healthy controls. However, oxyhemoglobin $\left(\mathrm{HbO}_{2}\right)$ showed a significantly faster rate of change in the healthy controls during the first 10 mins of the tilt and during the recovery. This NIRS muscle oximetry tool provides quantitative measurements of blood oxygenation monitoring in diseases such as POTS.
\end{abstract}

Keywords: Hemoglobin; head-up tilt table; oxygenation; blood volume.

$\S_{\text {Corresponding author. }}$

This is an Open Access article published by World Scientific Publishing Company. It is distributed under the terms of the Creative Commons Attribution 4.0 (CC-BY) License. Further distribution of this work is permitted, provided the original work is properly cited. 


\section{Introduction}

Postural orthostatic tachycardia syndrome (POTS) is a type of chronic orthostatic intolerance, annually affecting around 500,000 young Americans..$^{1,2}$ Symptoms of POTS include lightheadedness and persistent increase in heart rate $(>30 \mathrm{bpm}$ in adults and $>40 \mathrm{bpm}$ in children) with upright body posture. ${ }^{1-3}$ Patients with POTS may experience many other symptoms such as fatigue, sweating, tremors, anxiety, heart palpitation and exercise intolerance. ${ }^{1-5}$ Assuming a recumbent or supine position typically improves symptoms, which may lead to a high level of functional disability. ${ }^{4,5} \mathrm{~A}$ wide range of medications and treatment strategies are available, mainly emphasizing cardiac volume expansion and exercise. $^{5}$

In this study, the lower muscle oxygenation of POTS patients was monitored to assess the effect of the postural change during a head-up tilt table (HUT). HUT is a standard test for patients with a potential POTS diagnosis to evaluate their response to postural change. During the HUT, blood pressure and heart rate are measured continuously. ${ }^{6}$ However, subjects with an increase in heart rate that meets the diagnostic criteria for POTS may have the same symptoms as those who do not, often termed "orthostatic intolerance". ${ }^{7,8}$ Thus, other physiological abnormalities may in fact be critical to the development of orthostatic symptoms, which could be detectable with a device such as nearinfrared spectroscopy (NIRS), which evaluates deeper aspects of muscle and brain physiology. As a first step, we aimed simply to evaluate whether subjects with POTS showed clear differences from healthy control subjects. An affirmative answer to this question would encourage further studies that might separate patients with POTS from those with orthostatic intolerance without POTS.

NIRS is a noninvasive optical technique that utilizes wavelengths in the near-infrared window $(700-1300 \mathrm{~nm})$ to determine the in vivo concentrations of chromophores. NIRS oximetry is a viable method for inexpensively monitoring oxygen consumption in muscles and has been widely used to examine hemodynamics of skeletal muscle. ${ }^{9-11}$ Previous studies using NIRS oximetry have reported impaired cerebral oxygenation of patients with POTS. ${ }^{6}$ However, the pathophysiology remains unclear and research is required to understand the underlying conditions that lead to POTS.
The custom-made device can monitor the change in concentrations of the chromophores oxyhemoglobin $\left(\mathrm{HbO}_{2}\right)$ and deoxyhemoglobin $(\mathrm{Hb})$ based on the measured optical densities at certain wavelengths $735 \mathrm{~nm}$ and $850 \mathrm{~nm} .{ }^{12,13}$ These wavelengths are the absorbance peaks of $\mathrm{Hb}$ and $\mathrm{HbO}_{2}$ in the near-infrared window. ${ }^{14} \mathrm{~A}$ third wavelength near the isosbestic point of $\mathrm{Hb}$ and $\mathrm{HbO}_{2}(805 \mathrm{~nm})$, where the absorption of the two chromophores is equal, was used to find the change in combined concentrations of $\mathrm{Hb}$ and $\mathrm{HbO}_{2} \cdot{ }^{15}$

In this study, the NIRS oximeter monitored the lower muscle oxygenation of 6 POTS patients as well as 6 control subjects in our neuroscience research center during HUT test, and the trends were compared to the results from a commercially available NIRS monitor which can only provide oxygenation change (Covidien INVOS 5100C). Muscle oxygenation can provide important information regarding blood circulation and muscle functionality. ${ }^{16}$ Determining the muscle oxygenation of POTS after a physical intervention could potentially aid in the development of diagnostics and treatments that would mitigate the adverse effects of POTS and facilitate the recovery of tachycardia. In this study, the key relationship between impaired muscle oxygenation and POTS is investigated.

\section{Materials and Methods}

\subsection{NIRS Oximetry device}

The custom-built NIRS oximetry system [Fig. 1(a)] includes a probe head connected to a control box that controls the electro-optic components of the probe and records the real-time data. The control box consists of LED drivers and instrumentation for the detectors. The control box communicates with a digital acquisition board (DAQ board from National Instruments) simultaneously in both analog and digital channels, which is connected to a computer with LabVIEW software (National Instruments) interface.

The probe can produce and detect the reflected signal of three separate wavelengths of light from the tissue, simultaneously. The probe head as shown in Fig. 1(b) consists of an LED light source and photodetectors module. The light source is one triple wavelength LED (L735/805/850/PD-35B32, Epitex, Kyoto, Japan). This LED has a plastic 


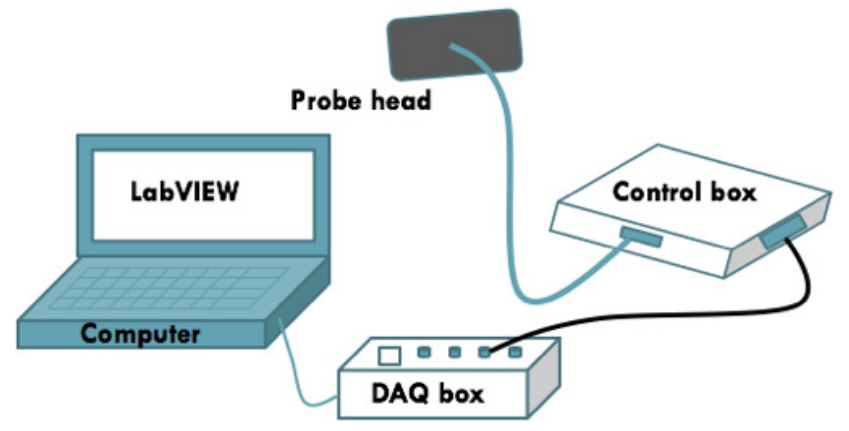

(a)

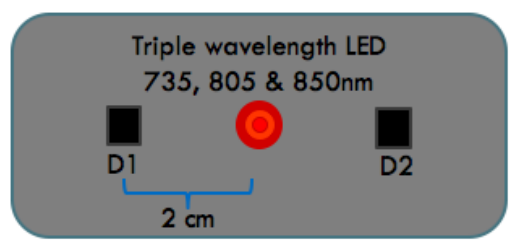

(b)

Fig. 1. (a) Custom-built NIRS Oximetry system consists of a control box and a probe connected to a laptop through a DAQ box. (b) The probe head geometry consists of a triple wavelength LED and two detectors, arranged in a line with a $2 \mathrm{~cm}$ source-detector separation.

Fresnel lens that collimates the beam. On either side of the source, two multi-pixel photon counters (S1133-14, Hamamatsu, Kyoto, Japan) are placed as detectors. These components are embedded in a silicone head to ensure that the distance between the source and detectors D1, D2 is fixed at $2 \mathrm{~cm}$.

\subsection{Monte Carlo simulation}

The optimal distance between the source and detector depends on the structure of the tissue, the wavelengths used and the desired depth of penetration. Increasing the source to detector separation results in a greater depth of penetration. ${ }^{10}$ However, increasing the separation distance decreases the signal-to-noise ratio. A modified Monte Carlo method is used to choose the optimal sourcedetector separation for the geometry of calf muscle oximetry.
The 3D Monte Carlo simulation is used to simulate photon distribution $(\phi)$ in a multi-layer heterogeneous model of the tissue. The tissue is modeled by $0.3 \mathrm{~mm}$ of the dermis, $1 \mathrm{~mm}$ of subdermis and a semi-infinite layer of the muscle beyond. Figure 2(a) illustrates Monte Carlo simulation results of light penetration in the tissue for $2 \mathrm{~cm}$ source to detector separation obtained by simulating the trajectories of $10^{8}$ photons through the tissue. Figure 2(b) shows the densest parts of these simulations to give a sense of the average optical path and the average depth of penetration. The $2 \mathrm{~cm}$ source-detector separation gives a depth of penetration up to $1 \mathrm{~cm}$.

\subsection{Data analysis}

In NIRS oximetry, near-infrared light is used to measure the changes in the concentration of $\mathrm{Hb}$ and

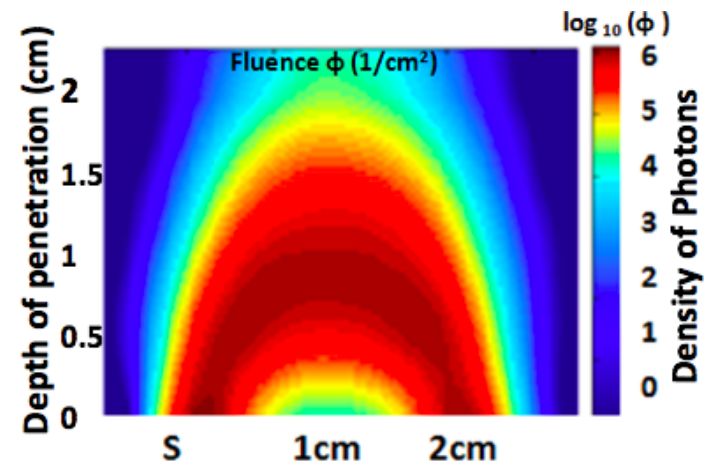

Source to detector distance $(\mathrm{cm})$

(a)

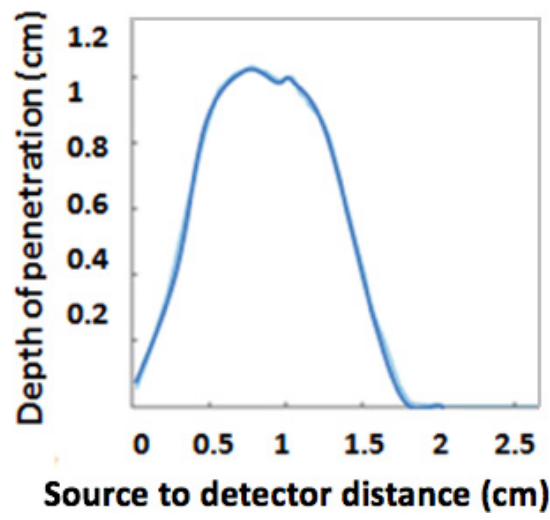

(b)

Fig. 2. (a) The photon propagation in Monte Carlo simulation for $2 \mathrm{~cm}$ source to detector separation. (b) The average effective photon path for the simulation. 
$\mathrm{HbO}_{2}$ via the modified Beer-Lambert law as

$$
\Delta \mathrm{A}=-\log \left(\frac{I(t)}{I_{o}}\right)=\Sigma L \mu_{\lambda_{i}} \Delta C_{i},
$$

where $\Delta \mathrm{A}$ refers to a change in attenuation, $I_{o}$ is the intensity of incident light and $I(t)$ is the intensity of detected light measured at some time $t . L$ is the average optical path length that the photons take from the source to the detector, $\Delta C_{i}$ is the change in concentration of the $i$ th given chemical and $\mu_{\lambda i}$ is the absorption coefficient of the given chemical at a certain wavelength equal to $\lambda$.

The three wavelengths can be used to calculate the amount of $\mathrm{HbO}_{2}$ and $\mathrm{Hb}$ by solving the following systems of linear regression equations:

$$
\left\{\begin{aligned}
\Delta \mathrm{A}(735 \mathrm{~nm})= & L\left(\mu_{\mathrm{Hb}}(735 \mathrm{~nm}) \Delta C_{\mathrm{Hb}}\right. \\
& \left.+\mu_{\mathrm{HbO}_{2}}(735 \mathrm{~nm}) \Delta C_{\mathrm{HbO}_{2}}\right) \\
\Delta \mathrm{A}(850 \mathrm{~nm})= & L\left(\mu_{\mathrm{Hb}}(850 \mathrm{~nm}) \Delta C_{\mathrm{Hb}}\right. \\
& \left.+\mu_{\mathrm{HbO}_{2}}(850 \mathrm{~nm}) \Delta C_{\mathrm{HbO}_{2}}\right) \\
\Delta \mathrm{A}(805 \mathrm{~nm})= & L\left(\mu_{\mathrm{Hb}}(805 \mathrm{~nm}) \Delta C_{\mathrm{Hb}}\right. \\
& \left.+\mu_{\mathrm{HbO}_{2}}(805 \mathrm{~nm}) \Delta C_{\mathrm{HbO}_{2}}\right)
\end{aligned}\right\},
$$

where $\Delta C_{\mathrm{Hb}}$ and $\Delta C_{\mathrm{HbO}_{2}}$ are the change in concentration over time. $\mu_{\mathrm{Hb}}(\lambda)$ and $\mu_{\mathrm{HbO}_{2}}(\lambda)$ are molar extinction coefficients of $\mathrm{Hb}$ and $\mathrm{HbO}_{2}$ at wavelength equal to $\lambda .{ }^{12}$ This system can be solved for three wavelengths to find the change in concentrations of $\mathrm{Hb}\left(\Delta C_{\mathrm{Hb}}\right)$ and $\mathrm{HbO}_{2}\left(\Delta C_{\mathrm{HbO}_{2}}\right)$.

The obtained changes in the concentrations of $\Delta C_{\mathrm{Hb}}$ and $\Delta C_{\mathrm{HbO}_{2}}$ are used to calculate the changein-oxygenation $(\Delta \mathrm{Oxy})$ and blood volume $(\Delta \mathrm{BV})^{16-18}$ as follows:

$$
\begin{gathered}
\Delta \mathrm{Oxy}=\Delta C_{\mathrm{HbO}_{2}}-\Delta C_{\mathrm{Hb}}, \\
\Delta \mathrm{BV}=\Delta C_{\mathrm{HbO}_{2}}+\Delta C_{\mathrm{Hb}} .
\end{gathered}
$$

Throughout the results section, negative $\Delta \mathrm{Oxy}$ implies that the muscle uses oxygen at a faster rate than can be supplied. Oximetry signals are acquired from the capillary bed that feeds the muscle. A decrease in $\Delta$ Oxy therefore reflects changes in both $\mathrm{HbO}_{2}$ and $\mathrm{Hb} .^{10,15}$

The averages of $\mathrm{HbO}_{2}, \mathrm{Hb}, \Delta \mathrm{Oxy}$ and $\Delta \mathrm{BV}$ were used both to make a quantitative oxygenation comparison between POTS patients versus healthy controls and for statistical analyses. The data were compared using the student two-tailed t-test with a significant difference criterion $p<0.01$. Linear correlation between our costume-built device and Covidien probe was evaluated in MS Excel.

\subsection{Subjects}

All the POTS patients and healthy controls were recruited under the approval of Institutional Review Board in the University of Wisconsin-Milwaukee and Medical College of Wisconsin (IRB\# 16.246, 558266). Six POTS patients (age 8-18 years) and six healthy control subjects (age $>18$ ) underwent the HUT protocol. Individual biometrics of these subjects is detailed in Tables 1 and 2 .

The POTS patients showed a rise in heart rate (HR) $>40 \mathrm{bpm}$ in first 10 mins of tilt as displayed in Table 3. The healthy control subjects were questioned before the procedure whether they were on any medications, or had no history of fainting, or lightheadedness with standing in the last year.

\subsection{Experimental protocol}

The NIRS oximetry probe was placed on the right gastrocnemius and a standard Covidien oximetry probe (INVOS 5100B, Covidien/Medtronic,

Table 1. POTS patient's data.

\begin{tabular}{lcccc}
\hline POTS patients & Age & Gender & Height $(\mathrm{cm})$ & Weight $(\mathrm{kg})$ \\
\hline 1 & 14 & $\mathrm{~F}$ & 175.7 & 58 \\
2 & 13 & $\mathrm{~F}$ & 157.5 & 41.1 \\
3 & 16 & $\mathrm{~F}$ & 168 & 63 \\
4 & 15 & $\mathrm{~F}$ & 159.9 & 75.3 \\
5 & 11 & $\mathrm{M}$ & 149.9 & 44 \\
6 & 16 & $\mathrm{~F}$ & 167 & 51.6 \\
\hline
\end{tabular}

Table 2. Healthy subject's data.

\begin{tabular}{lcccc}
\hline Healthy subjects & Age & Gender & Height $(\mathrm{cm})$ & Weight $(\mathrm{kg})$ \\
\hline 1 & 22 & M & 170.6 & 64 \\
2 & 21 & M & 173 & 65 \\
3 & 18 & F & 145.3 & 51 \\
4 & 22 & F & 158.9 & 60 \\
5 & 21 & F & 155.4 & 55 \\
6 & 23 & M & 182.8 & 63 \\
\hline
\end{tabular}

Table 3. Subject's heart rate changes.

\begin{tabular}{lccc}
\hline $\begin{array}{l}\text { Subject } \\
\text { type }\end{array}$ & $\begin{array}{c}\text { Max average HR } \\
\text { during baseline }\end{array}$ & $\begin{array}{c}\text { Max HR during } \\
\text { tilt (bpm) }\end{array}$ & $\begin{array}{c}\text { Tilt } \\
\text { duration } \\
\text { (mins) }\end{array}$ \\
\hline POTS & 76 & 123 & 30 \\
Healthy & 66 & 85 & 30 \\
\hline
\end{tabular}




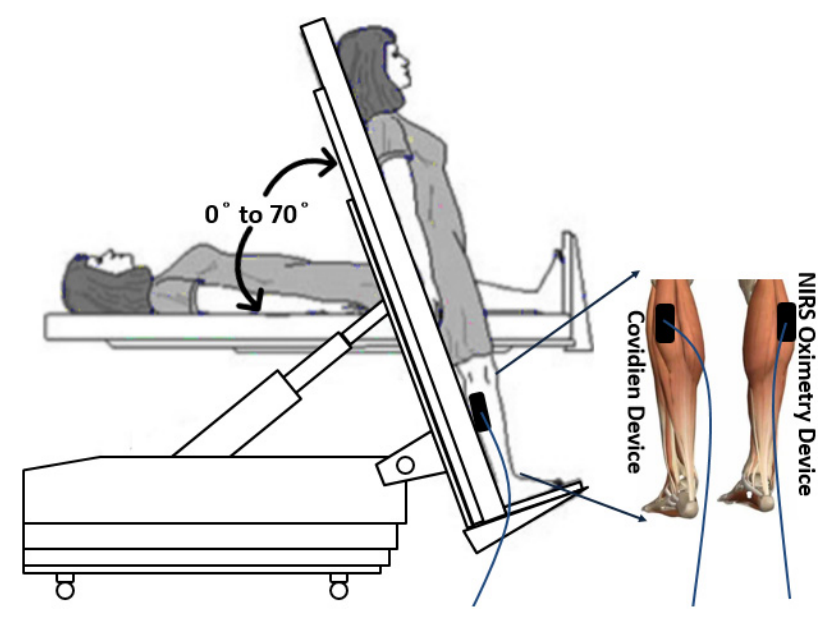

Fig. 3. The head-up tilt table (HUT) protocol; NIRS oximetry probe and Covidien probe are attached to the gastrocnemius muscle. The subjects were monitored at $0^{\circ}, 70^{\circ}$ and back to $0^{\circ}$ angles of the tilt table for $10 \mathrm{mins}$ at baseline, for $30 \mathrm{mins}$ at a $70^{\circ}$ tilt and for $10 \mathrm{mins}$ at recovery.

Minneapolis MN, USA) was placed on the left gastrocnemius to be used as a reference for comparison of $\Delta$ Oxy measurements.

The subject was asked to lay on the tilt table, monitored for 10 mins at $0^{\circ}$ angle of the HUT as the baseline. Monitoring continued for $30 \mathrm{mins}$ after they were taken to $70^{\circ}$ over $5 \mathrm{~s}$, and reclined to $0^{\circ}$ for $10 \mathrm{mins}$ to assess their recovery as shown in Fig. 3.

\section{Results}

\subsection{Validation of device}

As a reference for our custom-built NIRS oximetry probe, the Covidien probe was used to cross-check the $\Delta$ Oxy. Figure 4 shows the data comparison of the $\Delta$ Oxy obtained from the custom-built NIRS probe device and the Covidien oximetry device for healthy and POTS subjects. Regional $\Delta$ Oxy parameter for Covidien oximetry device is "percentage" (\%), whereas for the NIRS probe device $\Delta$ Oxy parameter is "change in concentration" (microMolar or $\mu \mathrm{M}$ ).

During the $70^{\circ}$ tilt, the data trend is similar to the $\Delta$ Oxy of both Covidien oximetry device and NIRS probe device for healthy subjects and POTS patients. The value of $R^{2}(0.95$ and 0.91$)$ shows the high correlation of the regression line between Covidien probe and custom-built NIRS oximetry probe.

\subsection{Physiological findings}

Figure 5 shows average $\mathrm{HbO}_{2}$ and $\mathrm{Hb}$ data collected from the healthy control subjects and patients with POTS. The three segments show the change in the posture of the subject from $0^{\circ}$ (baseline) to $70^{\circ}$ (tilt) and back to $0^{\circ}$ (recovery).

The $\mathrm{HbO}_{2}$ slightly increased during the $70^{\circ}$ tilt in healthy subjects and $\mathrm{Hb}$ increased during the same time. For POTS patients, the direction was similar, i.e., increase in $\mathrm{Hb}$ and a slight increase in $\mathrm{HbO}_{2}$, but the rise in $\mathrm{Hb}$ concentration in POTS patients was significantly greater than the rise in healthy subjects (see Fig. 6 for statistics).

The rates of change in $\mathrm{HbO}_{2}$ and $\mathrm{Hb}$ were analyzed during the 10 mins after the tilt was initiated and the subject was returned to a supine position. A significant difference in the rate of change of $\mathrm{HbO}_{2}$ was observed between the POTS patients and

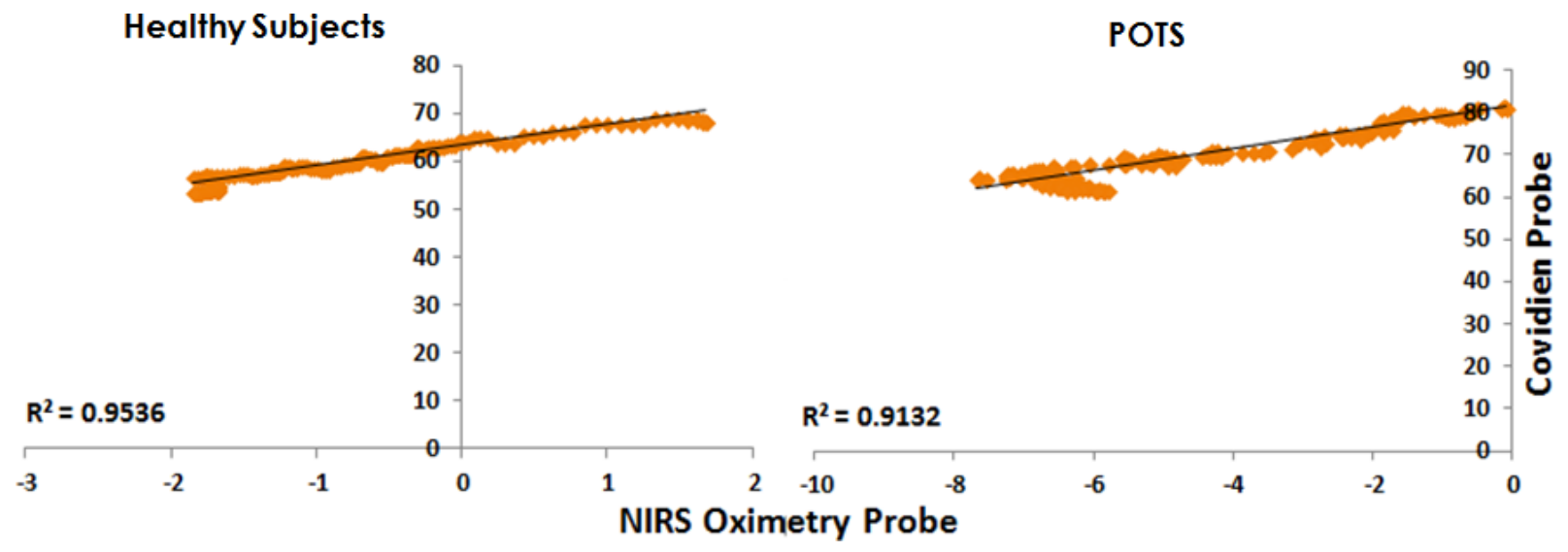

Fig. 4. Regional $\Delta$ Oxy correlation graph between Covidien probe and NIRS oximetry probe results in POTS and healthy subjects during the tilt at $70^{\circ} . R^{2}$ on the graphs closer to 1.0 show the better fit of the regression line. 
Oxyhemoglobin

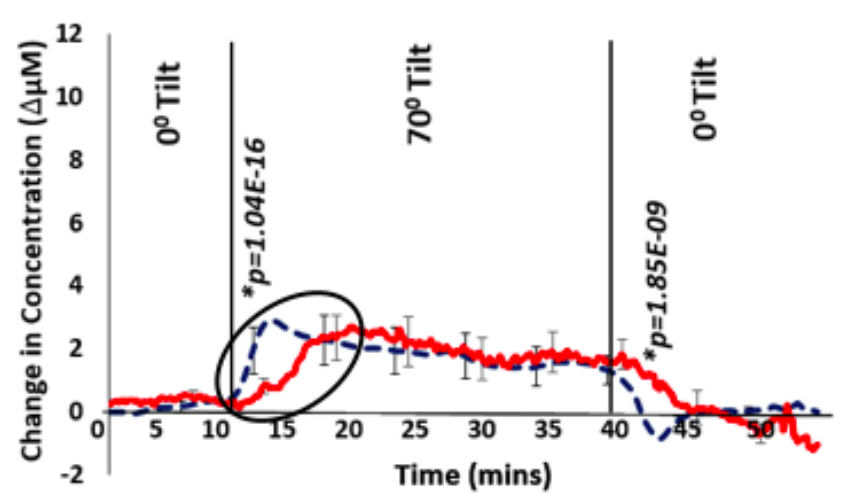

$--\cdot$ Healthy controls
Deoxyhemoglobin

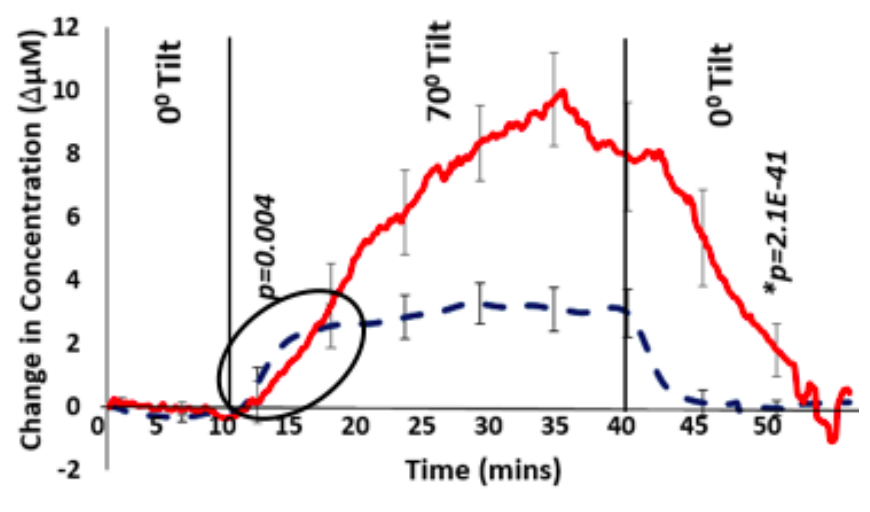

-POTS patients

Fig. 5. Data comparison between healthy subjects and POTS patients depicts changes in average $\mathrm{HbO}_{2}$ and $\mathrm{Hb}_{\mathrm{b}}$ concentrations on changing the position of the tilt table at $0^{\circ}, 70^{\circ}$ and $0^{\circ}$ for $10 \mathrm{mins}, 30$ mins and 10 mins, respectively. POTS subjects displayed a significantly higher increase in $\mathrm{Hb}$, while the change in $\mathrm{HbO}_{2}$ was statistically similar in both groups. The $p$-value $(p<0.01$ significant difference) was determined between the rising and dropping slopes of both the groups for $\mathrm{HbO}_{2}$ and $\mathrm{Hb}$.

healthy control group. The rates of change were tabulated and tested for significant difference using a two-tail Student t-test. This test resulted in significant difference $(p<0.01)$ rate of change in $\mathrm{HbO}_{2}$ immediately after the tilt was initiated and after it was stopped. $\mathrm{Hb}$ only showed significant difference after the tilt was ended. In Fig. 5, the $p$-values for comparing the rates were also presented.

The area under the curve (units: $\Delta \mu$ M.mins) was calculated during the 30 mins of tilt. The average area under the $\mathrm{HbO}_{2}$ curve in POTS was 42 and in healthy controls was 41 . The average area under the Hb curve in POTS was 113 and in healthy controls was 52 . The $\mathrm{HbO}_{2}$ curve area did not vary significantly, while the area under Hb for POTS was double the area under the healthy controls $\mathrm{Hb}$ signal.

\subsection{Statistical analysis}

In Fig. 6, each bar represents the averages of the $\mathrm{HbO}_{2}, \mathrm{Hb}, \Delta \mathrm{Oxy}$ and $\Delta \mathrm{BV}$ for both groups of POTS and healthy subjects. The values represent the mean $\pm \mathrm{SE}$ during the $70^{\circ}$ tilt.

$\mathrm{HbO}_{2}$ between patients and healthy controls did not show a statistically significant difference.

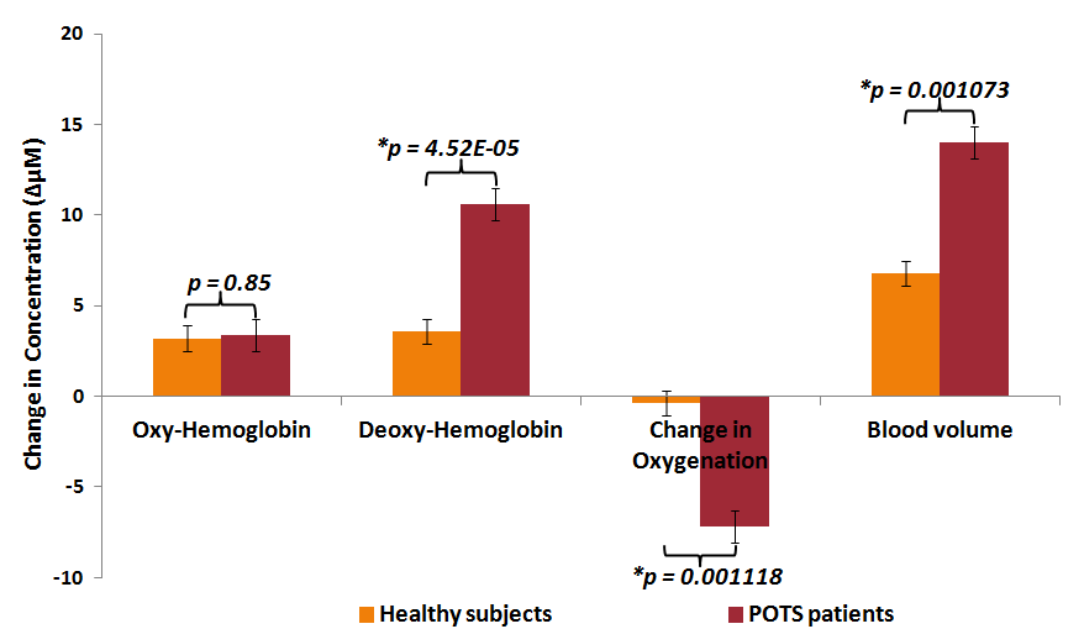

Fig. 6. Statistical analysis of healthy subjects and POTS patients. The two groups were compared in average $\mathrm{HbO}_{2}$, average $\mathrm{Hb}_{\text {, }}$ $\Delta \mathrm{Oxy}$ and $\Delta \mathrm{BV}$ during the $70^{\circ}$ tilt. Referring $p$-value $<0.01$ shows that there was a significant difference between healthy and POTS subjects $(n=6$ per group). 
$\mathrm{Hb}$ showed a significant difference between patients and healthy subjects. The observed difference showed that the Hb signal was significantly higher in patients with POTS than in healthy controls, while the two groups had a similar $\mathrm{HbO}_{2}$ level in their muscle.

$\Delta$ Oxy between patient and healthy controls showed a significant difference. The drop of $\Delta \mathrm{Oxy}$ concentration in patients was significantly greater than in healthy control subjects. Also, $\triangle \mathrm{BV}$ changes in POTS patients was significantly more than healthy controls.

\section{Discussions and Conclusions}

This study investigated the potential use of NIRS for the quantitative measurement of calf muscle oxygenation in POTS patients. Using noninvasive NIRS of the calf muscle, $\mathrm{HbO}_{2}$ and $\mathrm{Hb}$ were measured and the $\Delta \mathrm{Oxy}$ and $\Delta \mathrm{BV}$ were calculated. The reflectance NIRS system used a differential form of the modified Beer-Lambert law to relate the change in concentration of each of the chromophores to the change in optical densities at each wavelength. The custom-built probe was designed to be used on muscle geometry applied to study the oxygenation change in the calf muscle of patients suffering from POTS during a change in posture with head-up tilt table. To the best of our knowledge, this study is the first to investigate the impact of POTS on muscle oxygenation impairment that ensues during the postural change.

This study could potentially help in determining whether tissue tonicity plays a role in the symptoms experienced by POTS patients. Such results could be used to quantitatively track the progress of a therapy in terms of changes in muscle oxygenation. Another application would be in POTS examinations, as a means of providing objective evidence of the degree of the syndrome, which adds a new dimension to what can be measured. It would be interesting to look at subjects who have orthostatic intolerance without POTS and determine if they also have the same abnormality.

The muscle oximetry results revealed that POTS patients and controls showed similar hemodynamics during $10 \mathrm{~min}$ baseline measurements (Fig. 5) explaining that without a postural change, there was no hemodynamic impairment between POTS affected patients and control subject. A similar trend observed at the end of the recovering period showed that even though POTS patients might have some hemodynamic impairment during the postural change, after assuming a supine position, their hemodynamics could eventually recover to their baseline. However, during the recovery period, the recovery of $\mathrm{HbO}_{2}$ and $\mathrm{Hb}$ were slower in POTS patients compared to controls suggesting either a delay in muscle oxygenation recovery of POTS patients or such magnitude of departure from normal that recovery takes significantly longer.

After the postural change to $70^{\circ}$ tilt, all subjects experienced an increase in both oxy and $\mathrm{Hb}$ because the postural change caused venous blood to pool in the lower extremities. The rate of change was about the same in all subjects, but the healthy controls did not increase after 4 mins in contrast to 30 mins in the POTS group (Fig. 6). The area under the $\mathrm{Hb}$ curve in POTS was twice than the healthy subjects curve area. That implies the increase in $\triangle \mathrm{BV}$ in POTS patients. The higher $\mathrm{Hb}$ in POTS patients during $70^{\circ}$ tilt could be because of both impaired blood flow and increased oxygen consumption. The impaired blood flow is consistent with some of the tachycardia symptoms such as a drop-in blood pressure in upper torso and head or cerebral hypo-perfusion. However, during the $70^{\circ}$ tilt, there was no significant difference in hemoglobin levels between the two groups suggesting that the rate of oxygen consumption in POTS patients were similar to healthy controls. These findings were compatible with venous pooling and poor veno-constriction, as generally hypothesized for POTS pathophysiology. ${ }^{3}$

The remarkable differences in the pattern of change in $\mathrm{HbO}_{2}$ and $\mathrm{Hb}$ may provide a new understanding of the underlying pathophysiology of POTS (Fig. 5). Healthy subjects showed a sharp rise in $\mathrm{HbO}_{2}$ that was quickly halted within the first 2 mins upright, while in POTS patients took about 10 mins to rise to the same level of $\mathrm{HbO}_{2}$. Thus, the rate of change in $\mathrm{HbO}_{2}$ differed markedly between the two groups. This finding suggests that the POTS subjects did not appear to stop accumulating $\mathrm{Hb}$ during the tilt, which is highly suggestive of venous pooling. After the tilt ended and the subjects reclined, a similar phenomenon was observed. During the 10 mins after the tilt, $\mathrm{Hb}$ trend in healthy controls decreased at a faster rate than it did in POTS subjects. The slower rate in POTS subjects could be explained by either having less oxygenated blood or having trouble clearing the blood from 
their extremities that collected due to venous pooling.

These differences suggest that there was some active initial critical process rapidly responding to the upright position in healthy subjects, with the reverse occurring after reclining, that failed in POTS patients. Possible explanations might include: (1) rapid accumulation of arterial blood in a tissue compartment such as arteriovenous (AV) shunts in muscle or skin beds on tilt-up (perhaps serving as a vasoconstrictive signal) and the reverse on recline in healthy subjects, which failed to occur in POTS patients; (2) noncompliant arteries of POTS subjects because they were already maximally constricted or dilated and therefore had minimal ability to store blood in this compartment in response to the upright posture; (3) absence of the presumed central nervous system reflex that produced this response (i.e., some issue with the baroreflex proper); (4) change in the fascial structure in or around the muscle beds, that negatively affected arterial compliance and prevented rapid expansion or constriction of vessels; and (5) oxygenation of the tissues in POTS was inadequate (compared to healthy subjects) and the increase in leg blood with standing was immediately extracted so that $\mathrm{HbO}_{2}$ did not rise.

The regional $\Delta$ Oxy as measured by Covidien probe INVOS $5100 \mathrm{C}$ also showed a precipitous decline during the tilt that mirrors the $\Delta$ Oxy values from the NIRS oximeter. The average $\Delta$ Oxy signal between the NIRS oximetry and Covidien device shows the similar trend in all the subjects presenting a significant correlation between the two devices. The study showed (1) expected increase in venous blood was presumably based on the increase in $\mathrm{Hb}$, (2) more interestingly, unexpected rapid upstroke of $\mathrm{HbO}_{2}$ was seen in healthy subjects and was absent in the POTS patients, suggesting a new explanation for POTS pathophysiology. The slopes of normal compliance confirm Stewart's group findings about normal venous compliance. ${ }^{19}$ In POTS patients, we found that the arterial system was less compliant with the inability to receive additional blood volume, explaining the slower response rates observed.

In conclusion, the custom-built NIRS showed a capacity to quantitatively analyze changes in $\mathrm{Hb}$ and $\mathrm{HbO}_{2}$ leading to new insights about POTS. A device with this ability would have many applications in the clinical settings for monitoring blood oxygenation in patients. Future work should aim to determine whether the calf muscle activation of POTS patients is comparable to that in the healthy controls. The hypotheses could be further explored by imaging the muscle in the upright compared to supine positions. Such results would help to determine whether tissue tonicity plays a role in the symptoms experienced by POTS patients.

\section{Acknowledgments}

The authors would like to thank all the subjects who participated in this research, particularly the POTS patients. This research would not be possible without their contributions. This work is supported by UWM RGI $101 \times 290$ (M. Ranji), the Bleser Endowed Chair in Neurology (H. Whelan), the Baumann Research Endowment (H. Whelan) and Advancing Healthier Wisconsin grant \#5520298 (T. Chelimsky-PI). These funding sources were not involved in the preparation of this article.

\section{Conflicts of Interest Declaration}

The authors declare that there are no conflicts of interest related to this article.

\section{References}

1. S. Dahan et al., "Postural orthostatic tachycardia syndrome (POTS) - A novel member of the autoimmune family," Lupus 25, 339-342 (2016).

2. S. R. Raj, "Postural tachycardia syndrome (POTS)," Circulation 127, 2336-42 (2013).

3. R. Freeman et al., "Consensus statement on the definition of orthostatic hypotension, neurally mediated syncope and the postural tachycardia syndrome," Clinical Autonomic Research 21, 69-72 (2011).

4. H. Abed et al., Diagnosis and management of postural orthostatic tachycardia syndrome: A brief review, J Geriatric Cardiol. JGC 9, 61-67 (2012).

5. J. M. Stewart et al., "Local vascular responses affecting blood flow in postural tachycardia syndrome," Am. J. Physiol. Heart Circ. Physiol. 285, H2749-56 (2003).

6. H. Tanaka et al., "Impaired postural cerebral hemodynamics in young patients with chronic fatigue with and without orthostatic intolerance," J. Pediatrics 140, 412-417 (2002).

7. A. Ojha et al., "Comorbidities in pediatric patients with postural orthostatic tachycardia syndrome," J. Pediatrics 158, 20-23 (2011). 
8. J. M. Stewart et al., "Pediatric disorders of orthostatic intolerance," Pediatrics e20171673 (2017), doi: 10.1542 /peds.2017-1673.

9. M. Niwayama et al., "A 200-channel imaging system of muscle oxygenation using $\mathrm{CW}$ near-infrared spectroscopy," IEICE Trans. Inf. Syst. 85, 115-123, 2002.

10. M. MasoudiMotlagh et al., "Monitoring hemodynamic changes in stroke-affected muscles using nearinfrared spectroscopy," J. Rehabil. Assis. Technol. Eng. 2, 2055668315614195 (2015).

11. G. Yu et al., "Time-dependent blood flow and oxygenation in human skeletal muscles measured with noninvasive near-infrared diffuse optical spectroscopies," J. Biomed. Opt. 10, 024027-02402712 (2005).

12. R. V. Maikala, "Modified Beer's Law-historical perspectives and relevance in near-infrared monitoring of optical properties of human tissue," Int. J. Ind. Erg. 40, 125-134 (2010).
13. T. Aoyagi, "Pulse oximetry: Its invention, theory, and future," J. Anesthesia 17, 259-266 (2003).

14. Y. Lin et al., "Noninvasive, low-noise, fast imaging of blood volume and deoxygenation changes in muscles using light-emitting diode continuous-wave imager," Rev. Sci. Instrum. 73, 3065-3074 (2002).

15. J. M. Schnettler, J. A. Wallace, "Pulse oximetry as a diagnostic tool of pulpal vitality," J. Endodontics 17, 488-490 (1991).

16. B. Chance et al., "Time-resolved spectroscopy of hemoglobin and myoglobin in resting and ischemic muscle," Anal. Biochem. 174, 698-707 (1988).

17. J. Truijen et al., "Orthostatic leg blood volume changes assessed by near-infrared spectroscopy," Exp. Physiol. 97, 353-361 (2012).

18. T. Hamaoka et al., "Quantification of ischemic muscle deoxygenation by near infrared time-resolved spectroscopy," J Biomed. Opt. 5, 102-106 (2000).

19. J. M. Stewart, "Pooling in chronic orthostatic intolerance," Circulation 105, 2274-2281 (2002). 